\title{
Low Cost Printer for DLP Stereolithography
}

\author{
Joško Valentinčič ${ }^{1,}{ }^{*}$ - Matej Peroša ${ }^{1}$ - Marko Jerman ${ }^{1}$ - Izidor Sabotin ${ }^{1}$ - Andrej Lebar1,2 \\ 1Faculty of Mechanical Engineering, University of Ljubljana, Slovenia \\ 2Faculty of Health Sciences, University of Ljubljana, Slovenia
}

\begin{abstract}
A general research direction of stereolithography based on digital light processing $(D L P)$ is to reduce the production time and to increase manufacturing accuracy. Compared to fused deposition modelling (FDM) machines, machines for DLP stereolithography are expensive and thus not available to a broad range of users as it is the case with FDM 3D printers. Comparing technologies, DLP stereolithography offers quicker and more accurate production. In this paper, performances of a low cost DLP stereolithographic printer is presented. Three main challenges are treated: an uneven illumination of commercial DLP projectors, a direction of illumination and a selection of optimal 3D printing parameters. Uneven illumination of DLP projector results in smaller usable working area and poor printing quality. The problem is solved by implementing an appropriate software mask, thus the product quality is not influenced by its position on a working table. The direction of illumination has a key role in DLP stereolithography. It is shown that constrained surface (illumination through a transparent bottom of the vat) gives better 3D printing accuracy compared to free surface (illumination of the photopolymer surface) stereolithography. To further improve the product quality, the optimal process parameters are determined. Using the Taguchi based surface response methodology optimal process parameters are defined and by using them, the deviation of the actual dimensions from the specified dimensions is less than $80 \mu m$.
\end{abstract}

Keywords: DLP stereolithography, software mask, illumination, surface response method

Highlights

- An illumination intensity on the whole projection surface can be achieved by using an appropriate software mask, but the required exposure time increases significantly.

- $\quad$ Using Taguchi based surface response methodology, optimal process parameters were defined: exposure time $16.5 \mathrm{~s}$, layer thickness $0.06 \mathrm{~mm}$ and time between two consecutive exposures $4.1 \mathrm{~s}$.

- A low cost DLP stereolithography printer is able to print part which actual dimensions deviate from the nominal dimensions in $x$ and $y$ direction for $30 \mu \mathrm{m}$ and $10 \mu \mathrm{m}$ respectively, and $80 \mu \mathrm{m}$ in $z$ direction.

\section{O INTRODUCTION}

Additive manufacturing (AM) is a formal expression for a process which belongs to a class of technologies used to be called rapid prototyping technologies (RP). Several other terms are, or used to be, in use as well: automated fabrication, freeform fabrication or solid freeform fabrication, layer-based manufacturing, stereolithography or 3D printing [1]. There are numerous ways to classify AM technologies, but recently accepted ISO/ASTM 52900 standard [2] classifies AM processes regarding type of material, principle applied for fusion or bonding, feedstock that is used for adding the material and machine architecture, i.e. how the material is brought together.

Stereolithography is a liquid-based AM process for 3D parts by curing a photosensitive polymer kept in a vat, thus the term vat photopolymerization process is often used. Various light sources are used for photopolymerization [1]. Shorter the light wavelength higher the curing rate. Controlled light irradiation induces a curing reaction, forming a highly cross-linked polymer. Compared to other polymerbased AM technologies such as the extrusion or jetting based processes, the stereolithographic process can produce parts with fine features and good accuracy by using various polymers [3].

In the last years, the resolution of digital light processing (DLP) projector has been significantly improved due to the use of new low cost digital micromirror devices (DMD). In contrast to other light sources, the use of DLP projector enables building the whole layer at the same time. A DLP stereolithography is used in various fields including medical applications, where biocompatible and biodegradable materials must be used [4] and [5], and oceanography [6] for a better understanding and restoration of fragile marine ecosystems.

Two types of DLP stereolithography systems are distinguished, namely free surface and constrained surface stereolithography. In free surface (also denoted as top-down stereolithography), the layer is cured on the photopolymer surface and the product is sunk in the liquid from the surface towards the bottom of the vat. On the other hand, in constrained surface (also denoted as bottom-up) stereolithography, the layer is cured through the optically clear bottom of the vat, and a non-stick layer so that the printed structure does not adhere to the substrate. Thus, the product grows up from the vat and out of the liquid. The 
constrained surface stereolithography offers several advantages over a free surface based system. The main one is that the curing of liquid resin is sealed from the oxygen-rich environment [7]. By eliminating the oxygen inhibition effect, the liquid photopolymer resin can be cured faster. The diffusion effects of oxygen were investigated to have significant effects on the size, shape and properties of parts fabricated by stereolithography [8]. However, a cured layer is sandwiched between the previous layer and the bottom surface of the vat. The solidified material may adhere strongly to the bottom of vat causing the object to break or deform when the build platform moves up from the vat during the building process [9].

The state-of-the-art is continuous liquid interface production (CLIP) where DMD is moved continuously over the area of the medium while the projected image is updated accordingly. CLIP is using an oxygen-permeable window below the ultraviolet image projection plane, which creates a "dead zone" (persistent liquid interface) where photopolymerization is inhibited between the window and the polymerizing part [10]. Such printers are already available on the market for around 250,000 $€$.

A printing resolution that can be achieved by DLP stereolithography depends on the size of micromirrors in DMD and an optical system. In general, higher printing resolution is easier to achieve on smaller printing area by the use of suitable optics, but the smallest feature size that can be produced depends on the resin, too. Kinetics of photoinitiated multi-vinyl polymerisations have been discussed in an extensive review in [11], but in practice much simpler equations are used to describe the polymerisation kinetics in the fabrication of structures by stereolithography [4]. They can be used to determine optimal process parameters for the given resin, but the rest of the influential parameters such as machine and DMD performances should be considered, too. Here, the established design of experiments (DOE) techniques can serve well.

A low cost desktop stereolithographic printer using a commercial DLP projector as a light source was built and tested. All material costs to build the printer were below $1,500 €$. The projector has only a small bandwidth in the optical spectrum close to ultraviolet light; the majority is in visible spectrum. Since photopolymerization is faster when photopolymer is illuminated by shorter wavelengths, one cannot expect high productivity using such light source in DLP stereolithography. Additionally, the luminance was not equal on the whole projected area. To improve printing accuracy, the equal luminance over all projection area was achieved by developing an appropriate software mask. Further on, the influence of illuminance from bottom and top of the photopolymer vat was examined, i.e. a constrained surface method and a free surface method. At last but not least, the optimal process parameters were defined by Taguchi based surface response methodology.

\section{EXPERIMENTAL SETUP}

All experiments were performed on custom made DLP stereolithographic printers. A printer utilizing the free from surface method is shown in Fig. 1 and printer utilising the constrained surface method is shown in Fig. 2. Both printers are using the same software and hardware. The NC-code is prepared by Creation Workshop (DataTree3D Ltd., USA) installed on a laptop computer. The computer is connected to Arduino Mega 2560 microcontroller which is further connected to Gecko drive (Geckodrive Inc., USA) and a stepper motor. A stepper motor is used to rotate the threaded spindle. The motor has resolution of $1.8^{\circ}$ and the spindle with a pitch of $2 \mathrm{~mm}$ is used, therefore the resolution of $Z$ stage is $0.01 \mathrm{~mm}$.

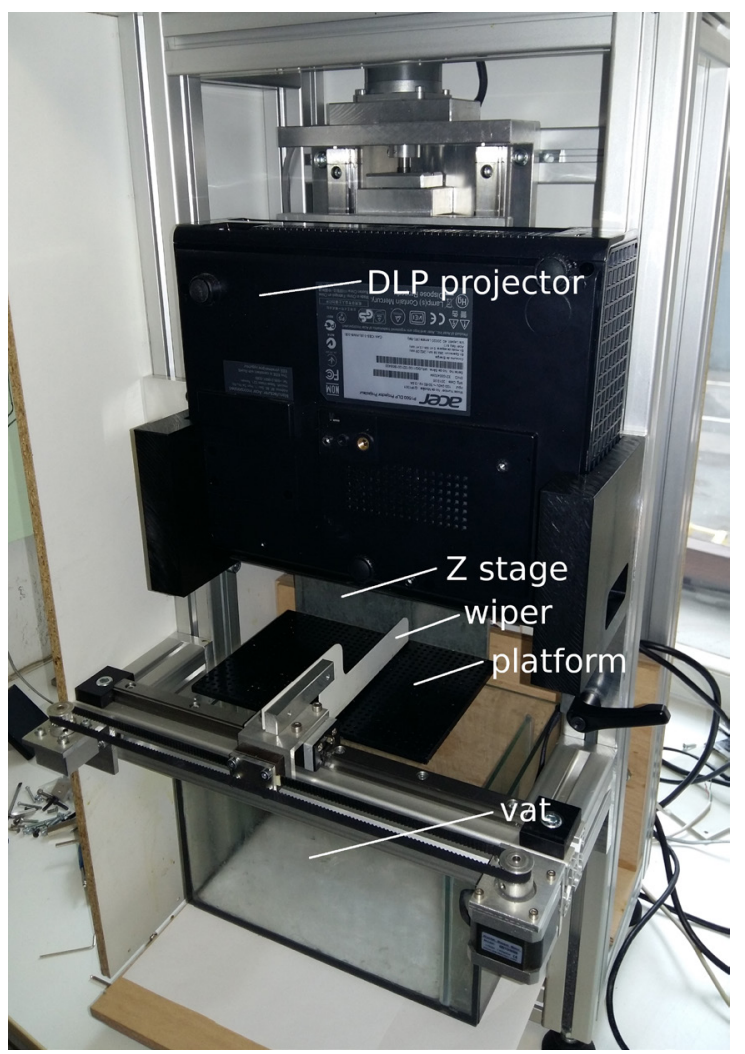

Fig. 1. A printer utilising the free surface printing method also denoted as top-down DLP stereolithography 


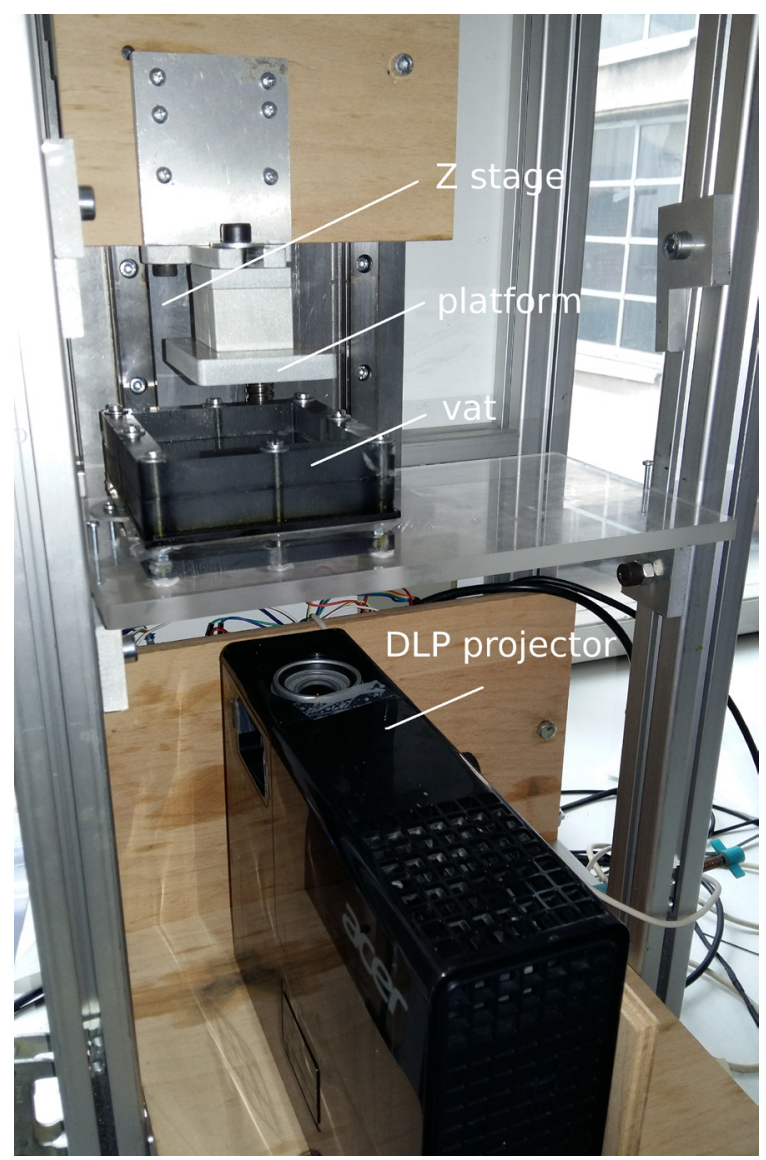

Fig. 2. A printer utilising the constrained surface printing method also denoted as bottom-up DLP stereolithography

In free form surface method, two gecko drives and two stepper motors are used. The second stepper motor is used to move mechanical wiper over the surface of photopolymer in order to avoid dipping and to redistribute the resin after curing each layer. In the case of constrained surface method, the mechanical wiper is not needed. In both cases, photopolymerisation was initiated by a DLP projector Acer P1500 having $1920 \times 1080$ pixels and 3000 lumen. The printing area was $88 \mathrm{~mm}$ by $50 \mathrm{~mm}$, thus the pixel size was $46 \mu \mathrm{m}$. The photopolymer used in this research was Deep Black (Fun To Do, Netherlands).

To build a software mask to compensate for uneven illumination, a testing site to capture the DLP projector image was build. The site consists of a computer connected to the DLP projector, a screen on which a white image was projected and a camera used to capture the image. The camera was a Canon $30 \mathrm{D}$ with $50 \mathrm{~mm}$ lens, $1 / 125 \mathrm{~s}$ shutter speed and $\mathrm{f} / 4.5$ aperture.

\section{COMPENSATION OF UNEVEN ILLUMINATION}

Commercial DLP projectors are not made to be used as a light source in DLP stereolithography, but with certain limitations they can do the job. The main drawback is the light spectrum. In preliminary tests of custom built DLP printer, it was observed that at certain locations of the printing area the test pieces were not entirely printed. The cause was the lack of uniformity in the DLP projector's illumination. The objective was to improve the printing area and printing quality by reducing the DLP projector's uneven illumination. The method, which is described in this article, uses a software mask to improve the DLP projector's illumination and printing quality. A mask is numerically applied over an image of projecting layer, thus the luminance of each pixel can be altered.

The projector was projecting the white light image to the screen and the picture of the image was taken by the camera. The screen had black border that was used to focus the camera (as shown in Fig. 3).

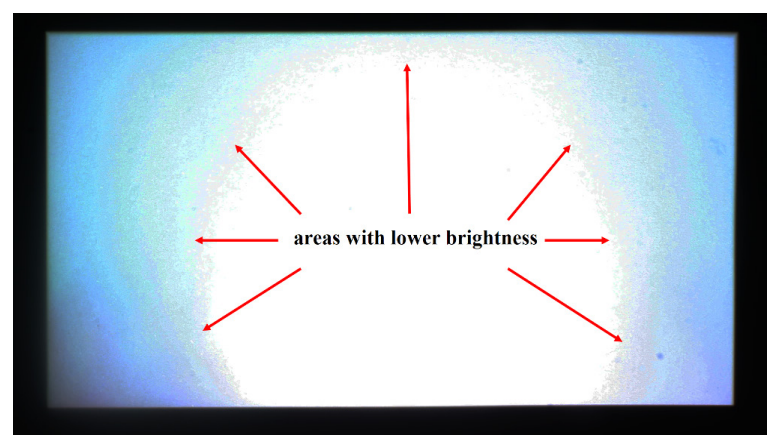

Fig. 3. A captured image of the DLP projector illumination with a size of $88 \mathrm{~mm}$ by $50 \mathrm{~mm}$; the image was modified for an easier presentation of areas with lower illumination

In the next steps the borders were cropped. The cropped image was then resized to $1920 \times 1080$ pixels and converted to a greyscale 8-bit PNG format, which is needed for image processing. The image was inverted i.e. the value of each pixel was subtracted from the brightest value which is 255 . The result was a rather dark image. Finally, a $50 \%$ transparency was set by changing the alpha channel value of each pixel on the dark image in Creation Workshop software. The result was a grey image that was used as a mask. The resizing, image conversion, image inversion and transparency setting were performed with a computer programme written in Microsoft Visual studio $\mathrm{C}++$. 


\section{DIRECTION OF ILLUMINATION}

A study was performed to find out which setup is better for the given printer: constrained surface method, i.e. building the part on the bottom of the vat, or free surface method, i.e. building the part on polymer surface. Both setups were evaluated by comparing the dimensions and visual inspection of test parts. In both cases the same focal length was used. In free surface method, the starting volume of the photopolymer in the vat was kept the same for all tests. When building product on the bottom of the vat, the photopolymer volume does not have a significant influence on the building process.

The test part given in Fig. 4 has external dimensions of $20 \mathrm{~mm} \times 22 \mathrm{~mm} \times 4 \mathrm{~mm}$. Five test parts were built by each method. Dimensions of several features were measured in $x, y$ and $z$ axis. Each measurement was repeated five times. Altogether, more than 1000 measurements were performed. All measurements were performed using camera with mounted appropriate lenses and custom made software developed in Matlab to measure the dimensions on recorded images.

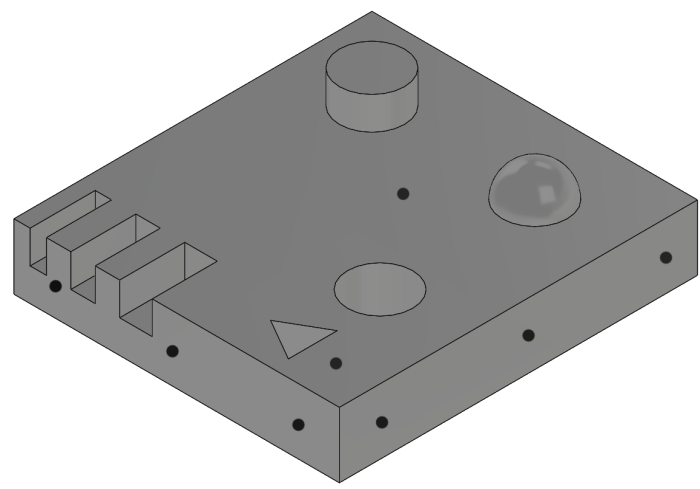

Fig. 4. Test part geometry with external dimensions $20 \mathrm{~mm} \times 22 \mathrm{~mm} \times 4 \mathrm{~mm}$; black dots indicate the measuring points in $x, y$ and $z$ direction

\section{PROCESS PARAMETERS}

DOE method is used to increase our understanding and knowledge of DLP stereolithography. For continuous improvement in product/process quality, it is fundamental to understand the process behaviour, the amount of variability and its impact on processes [12].

Based on preliminary tests, three printing parameters were selected, namely exposure time, layer thickness and time between the consecutive exposures with their maximum $(+1)$, mid $(0)$ and minimum $(-1)$ values. Thus, three parameters (factors) on three levels were used for the design of experiments. The factors and their values are shown in Table 1.

An $L_{18}\left(\begin{array}{ll}2^{1} & 3^{7}\end{array}\right)$ orthogonal array was chosen for DOE by the Taguchi based surface response methodology. The orthogonal array allows the design of experiments of one factor at two levels and seven factors at three levels. The factors that were not needed were removed with the help of a linear graph of the orthogonal array. The $L_{18}$ requires 15 experiments to be conducted.

Table 1. Process parameters and their levels

\begin{tabular}{lccc}
\hline \multicolumn{1}{c}{ Factor } & \multicolumn{3}{c}{ Level } \\
\cline { 2 - 4 } & -1 & 0 & +1 \\
\hline A: Exposure time [s] & 8.5 & 12.5 & 16.5 \\
\hline B: Layer thickness [mm] & 0.01 & 0.06 & 0.1 \\
\hline $\begin{array}{l}\text { C: Time between two } \\
\text { consecutive exposures [s] }\end{array}$ & 3.6 & 4 & 4.4 \\
\hline
\end{tabular}

The experiments were carried out in random order. In preliminary tests it was noted that the residence time of the photopolymer in the vat influences the quality of the part, thus the photopolymer was well shaken before it was poured into the vat. After each test, the vat was emptied and cleaned. After each test the printed test sample was measured using a micrometer.

The test sample has several features that served for visual inspection. It was noticed that visually good parts have also good dimensional accuracy, thus only the measured dimensions were used in optimization procedure. The dimensions in $x, y$ and $z$ directions were measured at the measuring points shown in Fig. 4. At each measuring point, the measurements were repeated three times.

\section{RESULTS AND DISCUSSION}

\subsection{Compensation of Uneven Illumination}

An image of the DLP projector illumination with the mask applied was captured and compared with the image without the mask. The results of the comparison are presented in Figs. 5 and 6.

For better visualisation, the intensity of white light is presented in colours. The discrepancy of white colour is significantly reduced when software mask is used as can be observed by comparing both figures.

It has to be noted that the intensity of the light by using mask is reduced, thus longer exposure time must be used and consequently the manufacturing time increases. The mask could be made to completely 
reduce discrepancy, i.e. the area in the lower left corner in Fig. 6 would not deviate from the rest of the image, but then the brightness of all pixels would equal to the brightness of the least bright pixel. Using such mask, the required exposure time would increase even more. In order to find the right balance between even illumination and exposure time, the unevenness cannot be completely eliminated.

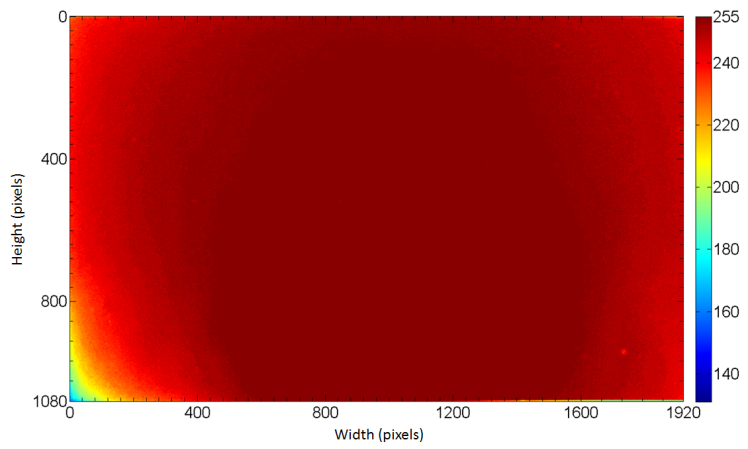

Fig. 5. Characteristics of DLP illumination without mask

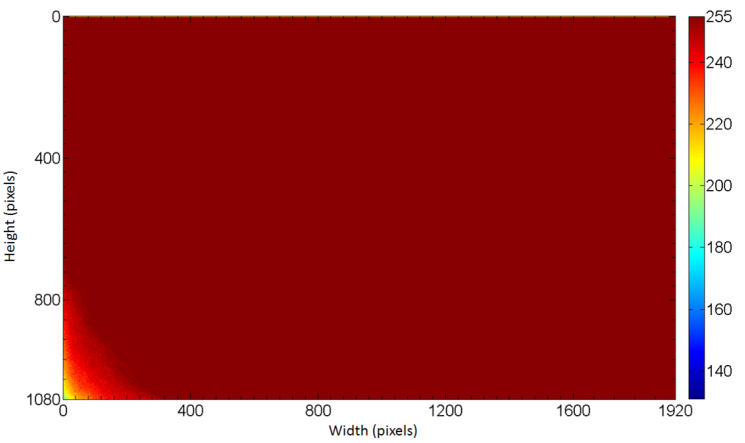

Fig. 6. Characteristics of DLP illumination with mask

\subsection{Direction of Illumination}

Two types of illumination were investigated, namely constrained surface method and free surface method. All together 1128 measurement data were collected and analysed separately regarding accuracy in $x$, $y$ and $z$ axis. Further on, measurements of inner dimensions are distinguished from measurements of outer dimensions. In $z$ axis only outer dimension measurements were performed. The average values are very close to nominal values (Table 2). One can notice that parts built by constrained surface method better meets the nominal values than the part built by free surface which is in agreement with the findings in literature [7] to [9], [13] and [14]. The same conclusion can be drawn when comparing standard deviations, which are gathered in the Fig. 7.
Although the average values of these dimensions are close to the nominal values (Table 2), standard deviations of dimensions around $0.07 \mathrm{~mm}$ in the case of constrained surface method (Fig. 7) indicate that the precision could be improved.

Table 2. Average deviations from nominal dimensions in $\mathrm{mm}$; where $A$ is a constrained surface method and $B$ is a free surface method

\begin{tabular}{lccccc}
\hline & \multicolumn{2}{c}{$x$ axis } & \multicolumn{2}{c}{$y$ axis } & \multirow{2}{*}{ z axis } \\
\cline { 2 - 5 } & outer & inner & outer & inner & \\
\hline A & 0.00 & 0.03 & -0.01 & 0.03 & -0.01 \\
\hline B & -0.14 & 0.16 & -0.18 & 0.08 & 0.31 \\
\hline
\end{tabular}

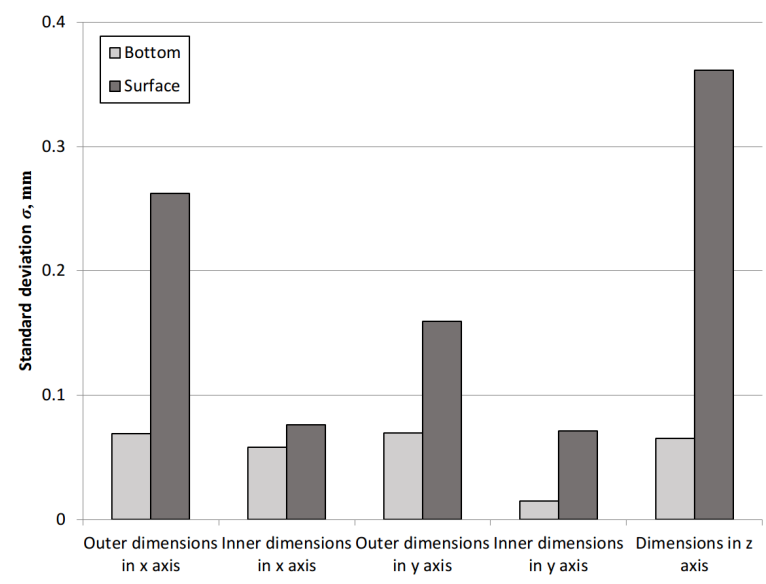

Fig. 7. Standard deviation of part dimensions; where OUTX is outer dimensions in $\mathrm{x}$ axis, INx inner dimensions in $x$ axis, OUTy outer dimensions in $y$ axis, INy inner dimensions in $y$ axis, and $\mathrm{z}$ dimensions in $\mathrm{z}$ axis

\subsection{Process Parameters Optimization}

Since constrained surface method gives better results than free surface method, only the former is further examined. According to $L_{18}\left(2^{1} 3^{7}\right)$ orthogonal array the experiments were performed as given in Table 3 , where the results of experiments are given, as well.

An analysis of the measurements is done according to the Taguchi based surface response methodology. The regression models are developed for each response, namely measured dimensions in $x, y$ and $z$ direction and are given in Eqs. (1) to (3) respectively.

$$
\begin{aligned}
x= & 22.04+5.57 \cdot A-6.49 \cdot B-10^{-3} \cdot C+2 \cdot 10^{-9} \cdot A \cdot B- \\
& -2 \cdot 10^{-9} \cdot A^{2}+20.57 \cdot B^{2}+10^{-7} \cdot C^{2} \\
y= & 21.55+3 \cdot 10^{-5} \cdot A-4.68 \cdot B+4 \cdot 10^{-5} \cdot C+ \\
& +10^{-4} \cdot A \cdot B=10^{-9} \cdot A^{2}+14.81 \cdot B^{2}
\end{aligned}
$$


$Z=4.72-8 \cdot 10^{-6} \cdot A-5.04 \cdot B-8 \cdot 10^{-5} \cdot C+$

$+22.22 \cdot B^{2}$

Table 3. Experimental values and results; where $A$ is exposure time, $B$ layer thickness and $C$ the time between two consecutive exposures

\begin{tabular}{rccccc}
\hline \multicolumn{3}{c}{ Factor } & \multicolumn{3}{c}{ Response } \\
\hline 8.5 & $B$ & $C$ & $x[\mathrm{~mm}]$ & $y[\mathrm{~mm}]$ & $z[\mathrm{~mm}]$ \\
\hline 12.5 & 0.01 & 3.6 & 20.16 & 21.91 & 4.17 \\
\hline 16.5 & 0.10 & 4.4 & 20.21 & 21.93 & 3.95 \\
\hline 8.5 & 0.01 & 4.0 & 20.15 & 21.90 & 4.41 \\
\hline 12.5 & 0.06 & 4.4 & 20.14 & 21.90 & 3.97 \\
\hline 16.5 & 0.10 & 3.6 & 20.12 & 21.89 & 3.97 \\
\hline 8.5 & 0.06 & 3.6 & 20.01 & 21.78 & 4.16 \\
\hline 12.5 & 0.10 & 4.0 & 20.07 & 21.84 & 3.97 \\
\hline 16.5 & 0.01 & 4.4 & 20.20 & 21.98 & 4.18 \\
\hline 8.5 & 0.10 & 4.4 & 19.99 & 21.77 & 3.97 \\
\hline 12.5 & 0.01 & 3.6 & 20.24 & 21.97 & 4.24 \\
\hline 16.5 & 0.06 & 4.0 & 20.15 & 21.93 & 4.00 \\
\hline 8.5 & 0.06 & 4.4 & 20.10 & 21.87 & 4.02 \\
\hline 12.5 & 0.10 & 3.6 & 20.10 & 21.86 & 4.01 \\
\hline 16.5 & 0.01 & 4.0 & 20.19 & 21.98 & 4.17 \\
\hline 8.5 & 0.10 & 4.0 & 19.98 & 21.75 & 4.07 \\
\hline 12.5 & 0.01 & 4.4 & 20.27 & 21.98 & 4.17 \\
\hline 16.5 & 0.06 & 3.6 & 20.11 & 21.87 & 4.12 \\
\hline & & & & &
\end{tabular}

The significance of the developed models is given by $F$-value, coefficient of determination $\left(R^{2}\right)$, adjusted coefficient of determination $\left(R_{\mathrm{adj}}{ }^{2}\right)$, predicted coefficient of determination $\left(R_{\text {pred }}{ }^{2}\right)$ and adequate precision $(A P)$. These values are given in Table 4.

Table 4. Significance of the models

\begin{tabular}{lccc}
\hline & \multicolumn{3}{c}{ Direction } \\
\cline { 2 - 4 } & $x$ & $y$ & $z$ \\
\hline$F$-value & 20.54 & 25.69 & 11.66 \\
\hline$R^{2}$ & 0.93 & 0.93 & 0.78 \\
\hline$R_{\text {adj }^{2}}$ & 0.88 & 0.89 & 0.71 \\
\hline$R_{\text {pred }}{ }^{2}$ & 0.78 & 0.83 & 0.57 \\
\hline$A P$ & 16.47 & 16.30 & 10.73 \\
\hline
\end{tabular}

The F-values of all models imply that the models are statistically significant. The predicted R-squared is in both cases in reasonable agreement with the adjusted R-squared (the difference has to be less than 0.2 ). The adequate precision estimates the signal to noise ratio, where a ratio greater than 4 is desirable. In our case, all models indicate an adequate signal and all models are valid. The models for dimension in $x$ and $y$ direction are highly significant whereas model for $z$ direction is still acceptable. A plausible cause could be
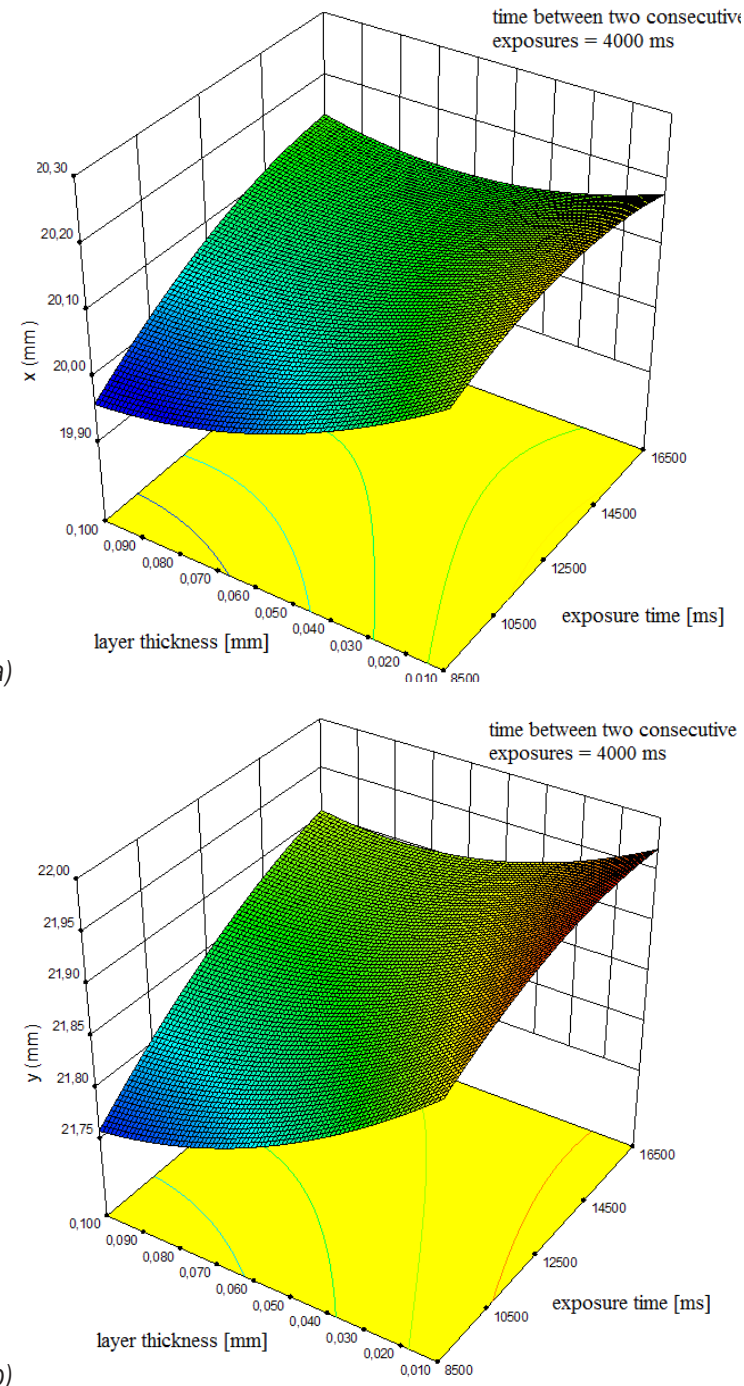

b)

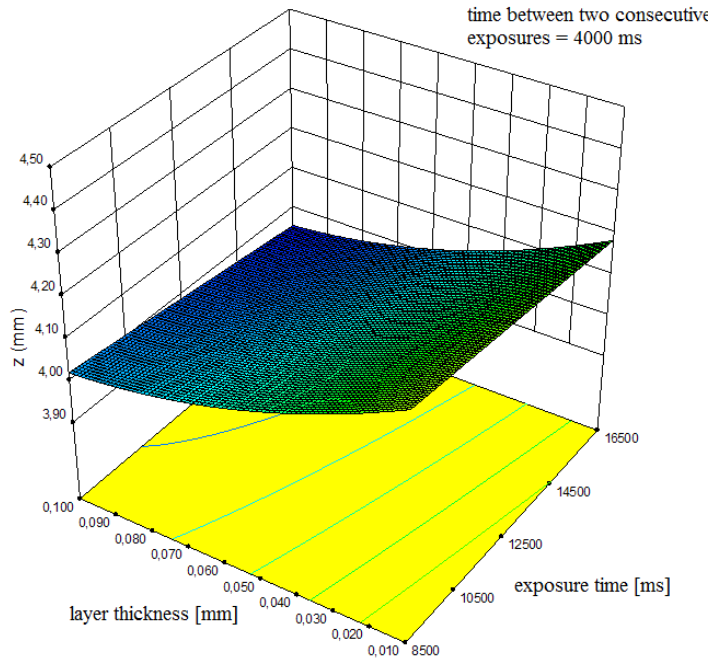

Fig. 8. Response surfaces; a) dimensions in x direction, b) dimensions in y direction, and c) dimensions in z direction, as a function of process parameters 
in the positioning of the platform. After each test the printing platform had to be separated from the printer to safely remove the test sample. When the platform was put back on its place, the positioning accuracy could not be compromised, thus the spacing between the printing platform and the bottom of the vat might not be the same as in the previous experiment trial.

The response surfaces are given in Fig. 8. One can notice that the dimensions in $x$ direction tend to be greater than the nominal value $(20 \mathrm{~mm})$ whereas the dimensions in $y$ axis tend to be smaller than the nominal one $(22 \mathrm{~mm})$. Thus, it is difficult to find process parameters that satisfy both requirements. This could be due to the misalignment of the projected image, the transparent bottom of the vat and the build platform which is mounted on the $\mathrm{z}$ stage.

After the analysis of the responses $x, y$ and $z$, a numerical optimization followed. The aim of the optimization is to determine the input parameters values in such a way that the responses are as close as possible to the nominal dimensions of the test sample $(x=20 \mathrm{~mm}, y=22 \mathrm{~mm}$ and $z=4 \mathrm{~mm}$ ). These are the three optimization criteria. The boundary condition is set as well: optimization is done within the limits of the input parameters values that were set during the planning phase of DOE.

The optimal input parameters, obtained with numerical optimization are: exposure time $16.5 \mathrm{~s}$, layer thickness $0.06 \mathrm{~mm}$, time between two consecutive exposures $4 \mathrm{~s}$. In the final phase, a series of confirmation tests were performed to verify whether the optimal parameters give the results that were predicted with the regression models.

Table 5. Results of confirmation tests and discrepancy between predicted and measured dimensions $(\Delta)$

\begin{tabular}{cccc}
\hline Response & Predicted $[\mathrm{mm}]$ & Measured $[\mathrm{mm}]$ & $\Delta[\mathrm{mm}]$ \\
\hline$x$ & $20.12 \pm 0.06$ & 20.15 & 0.03 \\
\hline$y$ & $21.91 \pm 0.04$ & 21.92 & -0.01 \\
\hline$z$ & $4.02 \pm 0.14$ & 3.94 & -0.08 \\
\hline
\end{tabular}

A total of three confirmation tests were performed and the report is given in Table 5. The predicted response values, i.e. dimensions of the part build by constrained surface method using the optimal parameters, lies within the determined confidence interval $(\alpha=0.05)$ and the dimensions of the part build by constrained surface method using the optimal parameters lies within the confidence interval. One of the test samples printed during the confirmation tests are shown in Fig. 9.

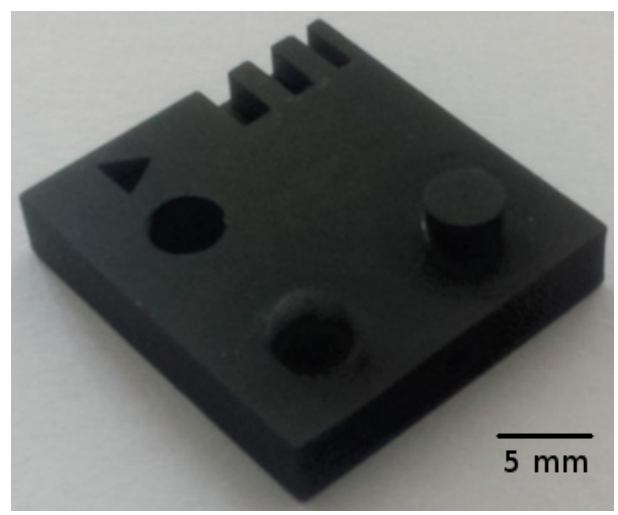

Fig. 9. Test sample manufactured for model confirmation test

\section{CONCLUSIONS}

Based on the results presented in this paper, the following conclusions can be drawn.

- The implementation of the mask successfully reduces the unevenness of the DLP projector's illumination and thereby the parts of the same quality are built in the entire printing area. But using the mask the exposure time increases significantly.

- A better printing accuracy is obtained when the photopolymer is illuminated through a transparent bottom of the vat, i.e. using the constrained surface method.

- The optimal printing parameters for the custom build 3D printer with applied software mask and photopolymer Deep Black were obtained: exposure time $16.5 \mathrm{~s}$, layer thickness $0.06 \mathrm{~mm}$, time between two consecutive exposures $4 \mathrm{~s}$. Using these parameters, the deviation of the actual dimensions from the nominal dimensions in $x$ and $y$ direction is $30 \mu \mathrm{m}$ and $10 \mu \mathrm{m}$ respectively, and $80 \mu \mathrm{m}$ in $\mathrm{z}$ direction.

- The material costs of the developed DLP stereolithography printer are less than $1500 €$. The printer is not suitable for professional work due to very long exposure time. A better illumination system with light spectrum closer to ultraviolet light and with even luminance over all projected area is needed, but then the stereolithographic printer is not a low cost machine any more.

With the illumination compensation and printing parameter optimization the printing quality of the prototype $3 \mathrm{D}$ printer was significantly improved, but there is still a few possibilities for improvements, e.g. to reduce the pixel size by reducing the area of illumination and to improve the parallelism between 
building stage, transparent bottom of the vat and the projected image.

\section{ACKNOWLEDGEMENTS}

The authors would like to thank to the Slovenian Research Agency for supporting the work in the frame of Research programme Innovative production systems (P2-0248).

\section{REFERENCES}

[1] Gibson, I., Rosen, D., Stucker, B. (2015). Additive Manufacturing Technologies, Springer, New York, DOI:10.1007/978-1-4939-2113-3.

[2] ISO/ASTM 52900:2015, Additive manufacturing - General principles - Terminology, International Organization for Standardization, Geneva

[3] Pan, Y., Chen, Y. (2016). Meniscus process optimization for smooth surface fabrication in Stereolithography. Additive Manufacturing, vol. 12, part B, p. 321-333, D0l:10.1016/j. addma.2016.05.004.

[4] Melchels, F.P.W., Feijen, J., Grijpma, D.W. (2010). A review on stereolithography and its applications in biomedical engineering. Biomaterials, vol. 31, no. 24, p. 6121-6130, DOl:10.1016/j.biomaterials.2010.04.050.

[5] Gauvin, R., Chen, J.-C., Lee, J.W., Soman, P., Zorlutuna, P., Nichol, J.W., Bae, H., Chen, S., Khademhosseini, A. (2012). Microfabrication of complex porous tissue engineering scaffolds using 3D projection stereolithography. Biomaterials, vol. 33, no. 15, p. 3824-3834, D0I:10.1016/j. biomaterials.2012.01.048.
[6] Mohammed, J.S. (2016). Applications of 3D printing technologies in oceanography. Methods in Oceanography, vol. 17 p. 97-117, D0l:10.1016/j.mio.2016.08.001.

[7] Pan, Y., Zhou, C., Chen, Y. (2012). A fast mask projection stereolithography process for fabricating digital models in minutes. Journal of Manufacturing Science and Engineering, vol. 134, no. 5, Dol:10.1115/1.4007465.

[8] Jariwala, A.S., Jones, H., Kwatra, A., Rosen, D.W. (2013). Process planning method for exposure controlled projection lithography. Proceedings of the $24^{\text {th }}$ Solid Freeform Fabrication Symposium, Austin, p. 95-110.

[9] Waheed, S., J.M. Cabot, N.P. Macdonald, t. Lewis, R.M. Guijt, B. Paull, M.C. Breadmore, (2016). 3D printed microfluidic devices: enablers and barriers. Lab on a Chip, vol. 16, no. 11, p. 1993-2013, D0I:10.1039/C6LC00284F.

[10] Tumbleston, J.R., Shirvanyants, D, Ermoshkin, N, Janusziewicz, R, Johnson, A.R., Kelly, D., Chen, K., Pinschmidt, R., Rolland, J.P.1, Ermoshkin, A., Samulski, E.T., DeSimone, J.M. (2015). Continuous liquid interface production of 3D objects, Science, vol. 347 , no. 6228 , p. 1349-1352, D0l:10.1126/science. aaa2397.

[11] Andrzejewska, E. (2001) Photopolymerization kinetics of multifunctional monomers. Progress in Polymer Science, vol. 26, no. 4, p. 605-665, Dol:10.1016/S0079-6700(01)00004-1.

[12] Antony, J. (2003). Design of experiments for engineers and scientists, Butterworth-Heinemann, Oxford.

[13] Huang, Y.-M., Kuriyama, S., Jiang, C.-P. (2004). Fundamental study and theoretical analysis in a constrained-surface stereolithography system. The International Journal of Advanced Manufacturing Technology, vol. 24, no. 5-6, p. 361369, D0I:10.1007/s00170-003-1627-9.

[14] Jariwala, A.S., Ding, F., Boddapati, A., Breedveld, B., Grover, M.A., Henderson C.L., Rosen, D.W. (2011). Modeling Effects of Oxygen Inhibition in Mask-based Stereolithography. Rapid Prototyping Journal, vol. 17, no. 3, p. 168-175, DOI:10.1108/13552541111124734. 